\title{
A Study on the Characteristics of EEG Signals by Auditory Stimulation of the Nature Sounds
}

\author{
Kwang-Bock You ${ }^{1}$, Zhixing Tian ${ }^{2}$ and Myung-Jin Bae ${ }^{3}$ \\ ${ }^{1}$ Soong-sil University, Department of Information and telecommunication Engineering, Seoul, 06978, Korea \\ Orcid Id : 0000-0002-3311-2418 \\ ${ }^{2}$ Soong-sil University, Department of Information and telecommunication Engineering, Seoul, 06978, Korea \\ Orcid Id : 0000-0003-3882-2459 \\ ${ }^{3}$ Soong-sil University, Department of Information and telecommunication Engineering, Seoul, 06978, Korea \\ Orcid Id : 0000-0002-7585-0400
}

\begin{abstract}
In this paper, the novel method that infers human emotions connecting to changes in brain waves is investigated. General methods or algorithms in this field of research extract the features from brain waves and use classifiers such as Support Vector Machine (SVM) and K-Near Neighbor (KNN), based on the artificial intelligence to explain human emotions. The novel method presented in this paper is to use a data table instead of involving the artificial intelligence-based classifiers of these common research methods. In order to prove the method proposed in this paper, the sounds of Niagara Falls, which is clearly nature sounds, recorded from near and far places are used as excitation signals to stimulate the brain. A simulation was performed to infer human emotions by analyzing and interpreting the brain waves obtained as a result of this experiment. The results of experiments and simulations performed in this paper fully demonstrate the method of extracting features from the measured EEG signals presented in this paper and inferring human emotions from the data table.
\end{abstract}

Keyword: Human Emotions, Brain Waves, SVM, KNN, Niagara Falls, Extracting Features, EEG

\section{INTRODUCTION}

A biological signal is a recording of biological events such as heartbeat or muscle contraction in space, time, or space-time. For this reason, the biological signals are used in medical diagnosis and in the understanding of the physiological mechanisms of any particular biological event or system. Among the biological signals produced by the human body, there is a brainwave signal that can explain human thoughts and emotions. The EEG signals, as well as voice signals, seem to be useful tools of enabling the so-called hyper-connection between people and people, people and objects or things, and humans and computers.

In this paper, the information collected from EEG signals is analyzed and interpreted so that human emotions corresponding thereto were investigated. Many studies that infer human emotions by analyzing EEG signals use Support Vector Machine (SVM) classifier and K-Near Neighbor (KNN) classifier widely exploited in the field of artificial intelligence research based on features that can be extracted from EEG signals. Fourier Information, Continuous and Discrete Wavelet Transform (CWT and DWT), Complex Wavelet Transform, Hilbert Transform, Hilbert-Huang Transform, and etc. are widely used as major features. In addition, as useful features that can be obtained in the spectrum domain, the parametric estimation spectrum by Autoregressive (AR) model and the Power Spectrum Density (PSD) function, obtained by Fourier transform of the autocorrelation function, are often used [1], [2].

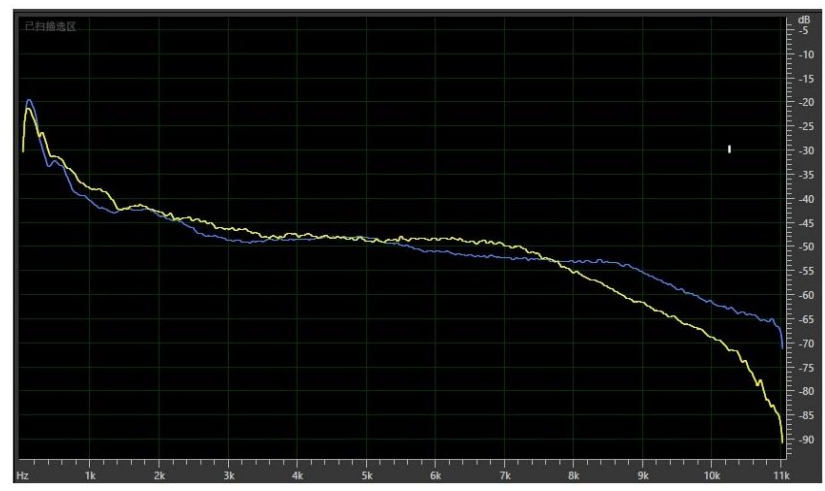

Figure 1. The Spectrum of Niagara Falls (yellow-far/ bluenear)

In this paper, the novel method that can infer human emotions without using classifiers such as SVM and $\mathrm{KNN}$ is proposed. In the frequency domain, for the EEG signals, the Fourier spectrum obtained by the Fourier transform and the estimated spectrum obtained through the AR Model were compared, analyzed, and analyzed. Then, the activity of the delta/ theta/ alpha/ beta/ gamma waves of the EEG signal responded by the excitation signal was exploited. The excitation signal used in this paper is a recorded sound from near and far from the sound of Niagara Falls, which is the nature sound. Figure 1 shows the spectrum of the excitation signal. This signal can be seen as white noise with energy distributed all over the frequency domain. According to many studies, white noise is helpful in sleep troubles. However, if this spectrum is analyzed in detail, this signal is closer to pink noise, which consists of all frequencies human beings can hear but the energy is not equally distributed across frequencies. In the spectrum of Figure 1, it can be seen that the energy is more intense than other bands at 0 to $1 \mathrm{kHz}$ of the low frequencies, which makes deep sleep. Of course, the energy of sound at far (yellow line) is decreasing sharply in $8 \mathrm{kHz}$ or higher than the energy of sound at near (blue 
International Journal of Engineering Research and Technology. ISSN 0974-3154, Volume 13, Number 8 (2020), pp. 1927-1933

(C) International Research Publication House. https://dx.doi.org/10.37624/IJERT/13.8.2020.1927-1933

line), but it can be said that it is basically the same [3], [4], [5]

Table 1. EEG Signals and Its Emotion States and Behaviors

\begin{tabular}{|c|c|c|c|c|c|}
\hline \multirow{2}{*}{$\begin{array}{l}\text { Brain } \\
\text { wave }\end{array}$} & \multirow{2}{*}{$\begin{array}{l}\text { Frequency } \\
\text { Ranges }\end{array}$} & \multirow{2}{*}{$\begin{array}{c}\text { State of } \\
\text { Consciousness }\end{array}$} & \multicolumn{2}{|c|}{ Mental or Physical State (Psychological State) } & \multirow{2}{*}{ Remark } \\
\hline & & & Active & (Weak) Non-Active & \\
\hline$\delta$ & $0 \sim 4 \mathrm{~Hz}$ & $\begin{array}{l}\text { Unconscious } \\
\text { level }\end{array}$ & Deep, Dreamless Sleep & $\begin{array}{c}\text { Inability to rejuvenate } \\
\text { body and to revitalize the } \\
\text { brain/ poor sleep }\end{array}$ & Negative emotion \\
\hline$\theta$ & $4 \sim 8 \mathrm{~Hz}$ & $\begin{array}{l}\text { Subconscious } \\
\text { level }\end{array}$ & $\begin{array}{l}\text { Dreaming/ Learning/ } \\
\text { meditation/ emotional } \\
\text { related behaviors }\end{array}$ & $\begin{array}{c}\text { Anxiety/ poor emotional } \\
\text { awareness/ stress }\end{array}$ & $\begin{array}{l}\text { Experiencing and } \\
\text { feeling deep and } \\
\text { raw emotions }\end{array}$ \\
\hline$\alpha$ & $9 \sim 13 \mathrm{~Hz}$ & $\begin{array}{l}\text { Conscious \& } \\
\text { Subconscious } \\
\text { level }\end{array}$ & $\begin{array}{l}\text { Relaxation/Engaging/ } \\
\text { Learning/ Thinking }\end{array}$ & $\begin{array}{l}\text { Anxiety/ high stress/ } \\
\text { insomnia }\end{array}$ & $\begin{array}{l}\text { Neutral emotion/ } \\
\text { Active activity of } \\
\text { the brain }\end{array}$ \\
\hline$\beta$ & $\begin{array}{c}14 \sim 30 \\
\mathrm{~Hz}\end{array}$ & $\begin{array}{l}\text { Conscious } \\
\text { level }\end{array}$ & Creativity & $\begin{array}{l}\text { Daydreaming/ } \\
\text { depression/ poor } \\
\text { cognition }\end{array}$ & Positive emotion \\
\hline$\gamma$ & $\begin{array}{c}31 \sim 100 \\
\mathrm{~Hz}\end{array}$ & $\begin{array}{l}\text { Conscious } \\
\text { level }\end{array}$ & $\begin{array}{l}\text { Concentration/ } \\
\text { Learning/ Reducing } \\
\text { stress }\end{array}$ & & Happiness \\
\hline
\end{tabular}

The classification of human emotions was based on the data collected from several papers and linked these results to the types of brain waves. Table 1 summarizes the EEG signals and their corresponding human emotions and behaviors with reference to [6], [7], [8], and [9]. The brain waves are defined in terms of the frequency bands and their state of consciousness is stated. The psychological state was classified when each brain wave was active and when it was non-active or weak. Finally, it was associated with possible emotional states.

Human emotions are usually classified into six sentiments: Happiness (Joy), Surprise, Anger, Fear, Sadness, and Disgust [10]. This paper adds the normal state to this classification and then re-classifies it into three categories: a positive sentiment such as happiness, joy, and surprise, and a negative emotion such as anger, fear, sadness, and disgust.

This paper consists of four sections. The introduction is presented in the section 1 . In the following section, the analysis of EEG signals is discussed. The Measurements and experiments are followed in the section 3 . In this section, both the data and the simulation results are analyzed. Finally, the conclusion of this paper is stat

\section{THE ANALYSIS OF EEG SIGNALS}

Interpreting the EEG signals as the sum of electrical signals by chemical activation between cells generated by brain activity makes it impossible to interpret very complex human thoughts and emotions. In order to interpret human thoughts and emotions, the processing and interpretation of EEG signals and several technologies are studied, and the technologies used in this paper are described.
In general, the probability distribution of multichannel EEG signals is considered as the multivariate Gaussian. Nevertheless, the statistical properties such as mean and covariance may vary for each analysis frame. Therefore, the EEG signal can be said to be stationary within the short-time analysis frame, and this is called Quasi-Stationary. These Gaussian assumptions are established while the human emotion is in the Normal State, but these assumptions are not valid if there is mental or physical activity. Thus, if the distribution of the EEG signal is measured at any "non-Gaussian" other than Gaussian, it means that it operates in a certain state. This "non-Gaussianity" can probably be measured by using the higher-order moments. For instance, there is the skewness as for 3rd moments and the Kurtosis as for 4th moments [1]. In addition, there are many analysis parameters and methods. The DWT, for instance, is used to extract the features from the EEG signal. By using the DWT, the single prototype function called the mother wavelet is used to decompose the input signal based on scaling and shifting parameters [1], [8]. Entropy, which uses to measure the randomness of signal, is a strong feature useful for analyzing the psychological time series data. The energy can be calculated by squared the wavelet coefficients of each sub-band [8], [11], [12]. These features are used to classify human emotions using the K-nearest neighbor (KNN) classifier and Supporting Vector Machine (SVM) classifier. Algorithms and methods using these classifiers are difficult to overcome with the computational burden and complexity of the hardware. Finally, there is a method of extracting features by applying the HilbertHuang Transform (HHT), which can perform a spectrum analysis of nonlinear and nonstationary data, to the EEG signal [13], [14], [15]. 
In this paper, the analysis in the frequency domain was focused and the following features were extracted and analyzed. First, the frequency spectrum was calculated by the following equation of the discrete Fourier transform.

$$
X(f)=\sum_{n=-\infty}^{\infty} x[n] e^{-j 2 \pi f n}
$$

This equation shows that a continuous frequency spectrum is obtained by performing the Fourier transform of a non-periodic discrete-time signal. This is very useful for signal analysis. However, it can be difficult to interpret as shown in Figures 3, 4 , and 5. Therefore, in this paper, the frequency spectrum was also analyzed using the Power Spectral Density (PSD) function and the Autoregressive (AR) processes model, which is a parametric estimation method. The following equation is the definition of the autocorrelation function (ACF) for the WideSense Stationary (WSS) Random signal.

$$
\begin{gathered}
R_{X}(k) \triangleq E[X(m+k) X(k)]=E[X(k) X(m+k)] \\
=R_{X}(-k)
\end{gathered}
$$

Signal $\mathrm{X}(\mathrm{m})$ is a discrete-time WSS random signal and the ACF is an even function. In addition, the PSD function is defined as the Fourier Transform of this ACF, which is expressed by the following equation.

$$
S_{X}(f) \triangleq \mathcal{F}\left\{R_{X}(k)\right\}=\sum_{k=-\infty}^{\infty} R_{X}(k) e^{-j 2 \pi f k}
$$

Since the ACF can be expressed with the Autocovariance function and its Mean.

$$
R_{X}(k)=C_{X}(k)+\left|m_{X}\right|^{2}
$$

Thus,

$$
S_{X}(f)=\mathcal{F}\left\{C_{X}(k)+\left|m_{X}\right|^{2}\right\}=\mathcal{F}\left\{C_{X}(k)\right\}+\left|m_{X}\right|^{2} \delta(f)
$$

The first term is the Fourier transform of the Autocovariance function and the second term represents the power at zero frequency which may say DC component of the signal. This justifies that DC components were removed from each EEG data that collected from the experiments.

Consider an AR model that can be expressed by the following equation.

$y(n)=\sum_{k=1}^{p} a(k) y(n-k)+x(n)$

Here, $y(n)$ are discrete-time WSS random sequences and $x(n)$ are the white noise sequences. In this equation, the coefficients $\mathrm{a}(\mathrm{k}), \mathrm{k}=1,2, \cdots, \mathrm{p}$ can be interpreted as predictor parameters. Thus, this equation can be interpreted that the value of current sample, $y(n)$ is as the sum of weighting the prediction coefficients, a(k) to the past samples, $y(n-1), \cdots, y(n-p)$ with adding the prediction error, $x(n)$. Therefore, the current sample is estimated as follows:

$$
\hat{y}(n)=\sum_{k=1}^{p} a(k) y(n-k)
$$

The prediction coefficients $\mathrm{a}(\mathrm{k})$ can be obtained from the following Yule-Walker equation by using, so called, minimizing the Mean Square Prediction Error (MMSE) methods.

$$
\begin{gathered}
{\left[\begin{array}{rlcc}
R(0) & R(-1) & \ldots & R(-p+1) \\
R(1) & R(0) & & R(-p+2) \\
& \vdots & \ddots & \vdots \\
R(p-2) & R(p-3) & & R(-1) \\
R(p-1) & R(p-2) & \cdots & R(0)
\end{array}\right]\left[\begin{array}{c}
a(1) \\
a(2) \\
\vdots \\
a(p-1) \\
a(p)
\end{array}\right]} \\
=\left[\begin{array}{c}
R(1) \\
R(2) \\
\vdots \\
R(p-1) \\
R(p)
\end{array}\right]
\end{gathered}
$$

Then, the following all pole transfer function of the AR (12) model to find the estimated spectrum is set:

$$
H(z)=\frac{1}{1-\sum_{k=1}^{p} a(k) z^{-k}}
$$

\section{MEASUREMENTS AND EXPERIMENTS}

\subsection{Data for Experiments and Simulation}

In this paper, EEG signals were collected from three testees who are in twenties. The sound recorded in the near (Near) and far (Far) sound of Niagara Falls shown in Fig. 1 was heard to each testee and then the EEG data was measured. Here, for the purpose of comparison, the EEG signal was measured even when nothing was heard. The experiment was conducted for about 2 minutes and 30 seconds while the testees were keeping the eyes closed and maintaining a quiet state. Then, DC components were removed from each EEG data that collected from the experiments. These signals were normalized using the mean and variance, and framing with the Hamming window with a length of 4 seconds, and then simulated with the data of this frame. Equation (10) is used for normalization. In this equation, $\boldsymbol{\mu}_{\boldsymbol{x}}$ and $\boldsymbol{\sigma}_{\boldsymbol{x}}$ are the sample mean and the sample standard deviation, respectively [16], [17], [18].

$$
\widehat{x(n)}=\frac{x(n)-\mu_{x}}{\sigma_{x}}
$$

The sampling frequency for the collected data is $256 \mathrm{~Hz}$. Since the meaningful frequency band in the EEG data is from delta wave to gamma wave over 0 to $100 \mathrm{~Hz}$, the sampling frequency of $256 \mathrm{~Hz}$ used in this paper is large enough by Nyquist Theorem [19].

\subsection{Experiments and Simulation Results}

Figure 2 shows the Isopotential Map of Testee1. In the Beforestate, $\delta$-wave is mainly seen. At the Near-state, $\alpha$ - wave is activated, and considerable amount of $\beta$-wave is shown. This seems to indicate that the brain recognized the sound and to somewhat a positive emotion. In the Far-state, it is much similar to the Near-state, but the $\alpha$-wave and $\beta$-wave were relatively decreased. 
The Isopotential Map in Figure 2 shows the change over the entire time of the data. For that reason, the short-time period analysis of data of Testee 2 and 3 was performed and its results were shown in Figures 3 through 8.
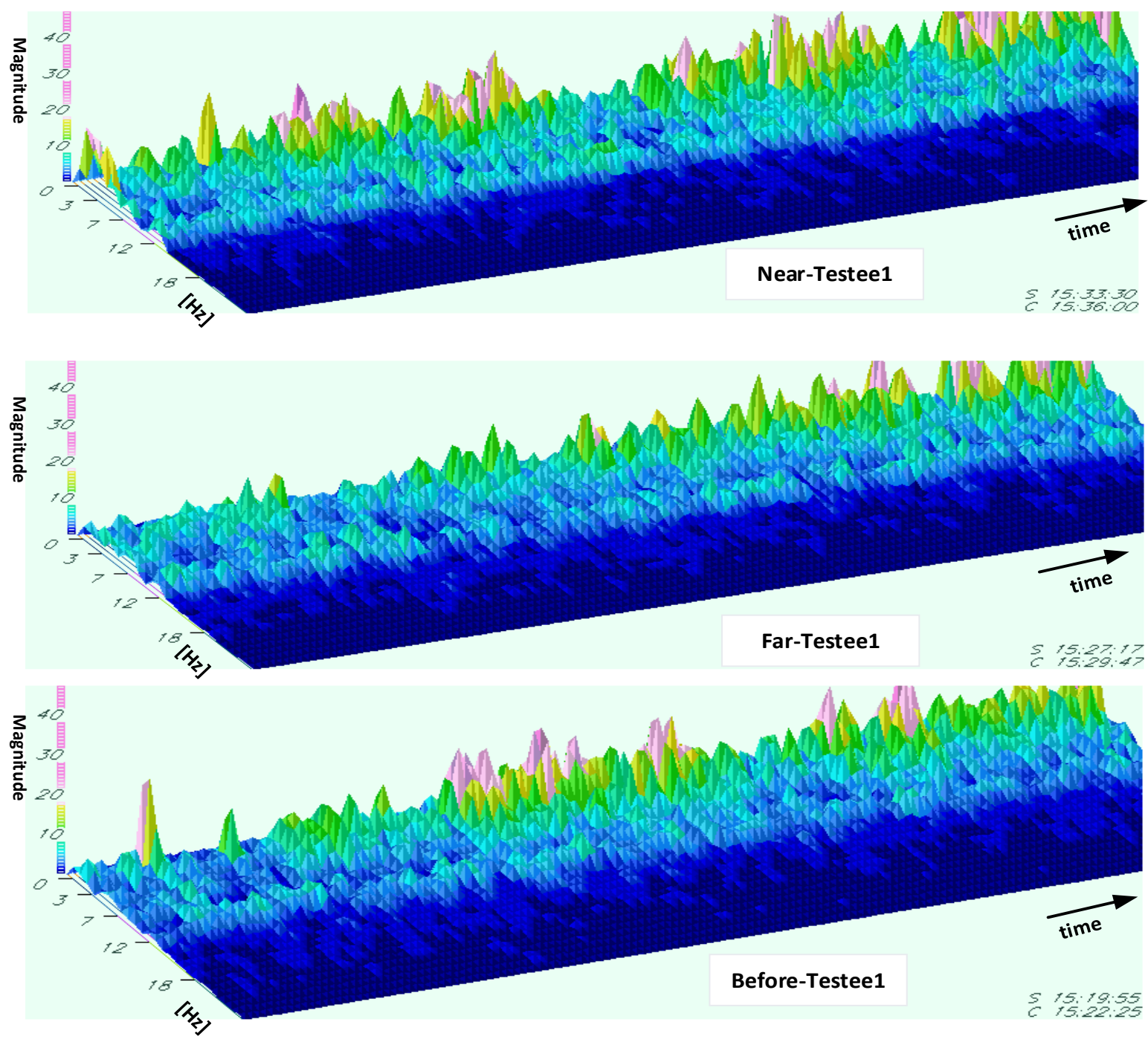

Figure 2. EEG Isopotential Map of Testee1 Niagara falls sounds - Near/ Far/ Before

In this paper, the short-time periods were set to 4 seconds. Each figure is composed of an EEG signal in the time domain, its Fourier Spectrum, Spectrogram, and PSD estimated by AR (12) model.
The EEG signals of Testee 2 were analyzed In Figures 3 to 5 . Compared to the Before-state, $\delta$-wave decreases in the Nearstate and the Far-state, while $\alpha$ and $\gamma$-wave increase, but there seems to be no big difference between the Before-state and the Near-state. 
International Journal of Engineering Research and Technology. ISSN 0974-3154, Volume 13, Number 8 (2020), pp. 1927-1933

(C) International Research Publication House. https://dx.doi.org/10.37624/IJERT/13.8.2020.1927-1933
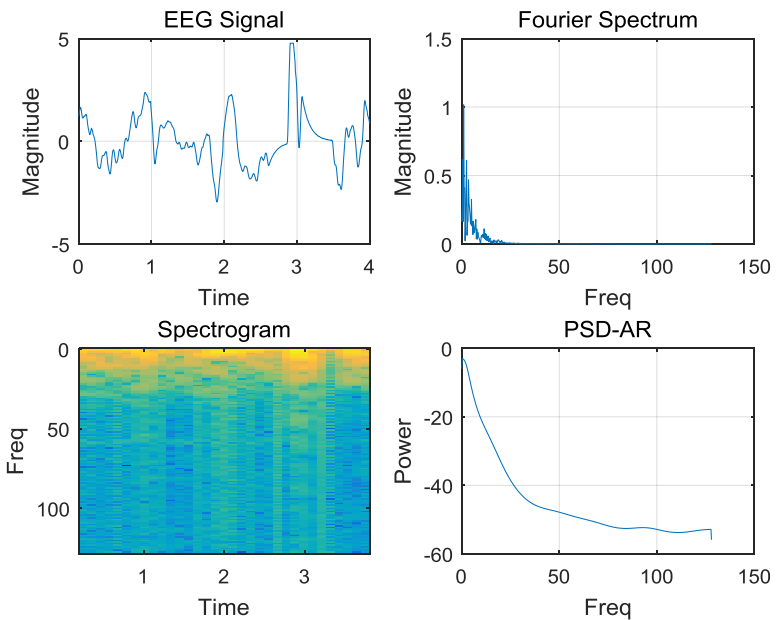

Figure 3. Testee2A-before
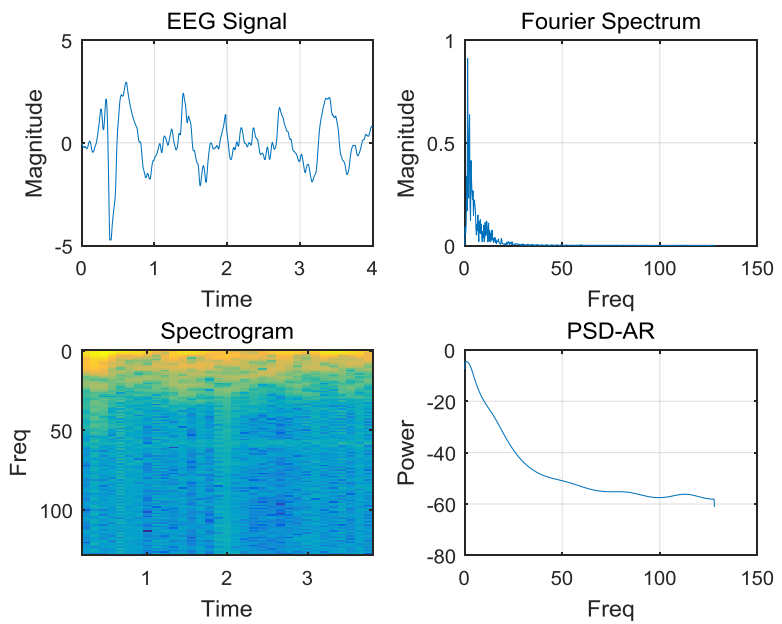

Figure 4. Testee2A-near
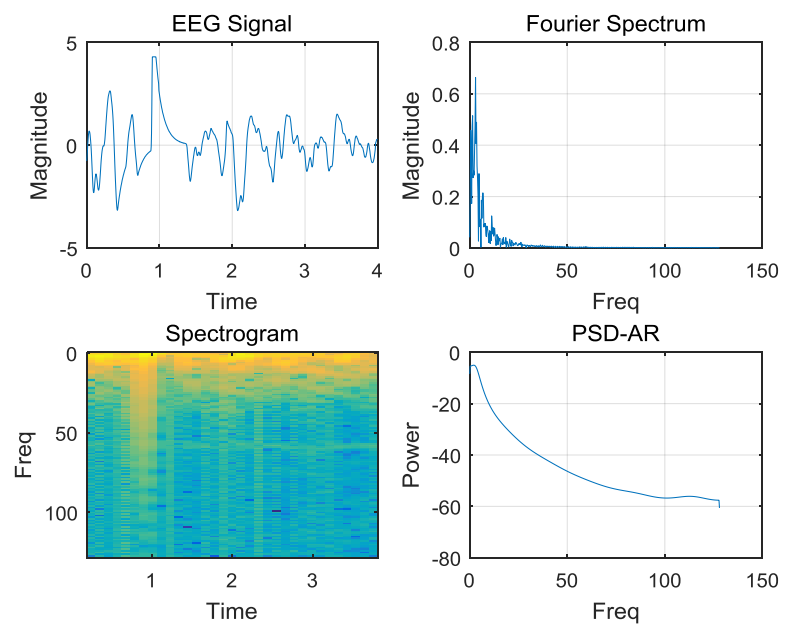

Figure 5. Testee2A-far
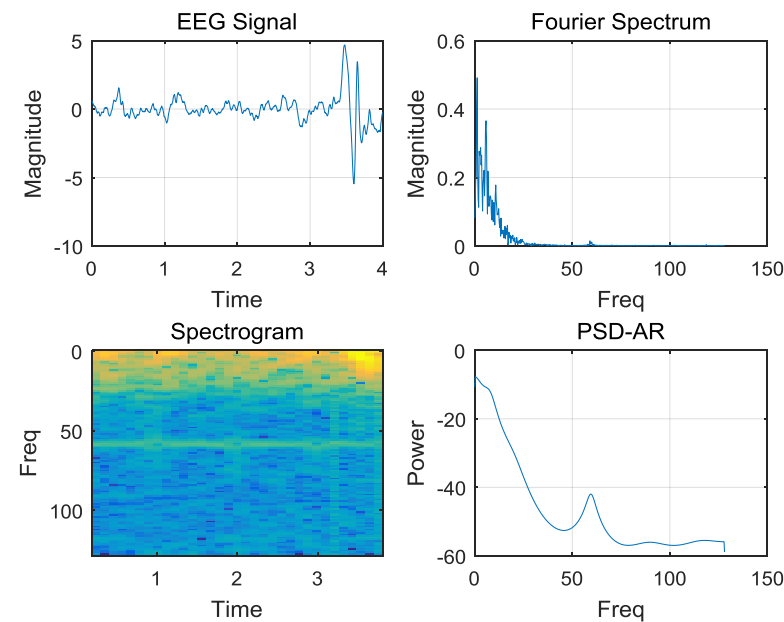

Figure 6. Testee3A-before
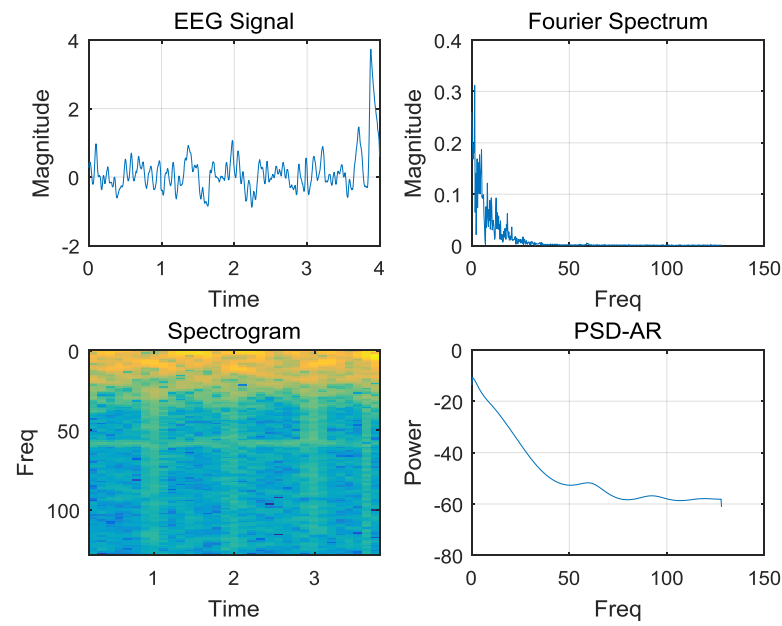

Figure 7. Testee3A-near
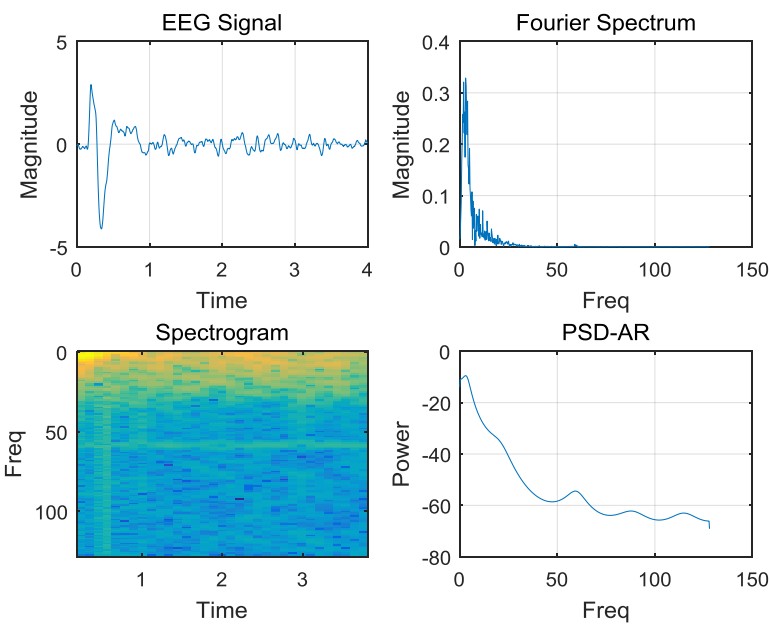

Figure 8. Testee3A-far 
In Figures 6 to 8, the EEG signals of Testee 3 were analyzed. A small mountain in the $\gamma$-wave region, which is difficult to see in the Fourier Spectrum of the Before-state, is clearly shown in PSD-AR (12). From the spectrogram, it can be seen that the energy distribution in the Near-state and the Far-state is considerably wider than that of the Before-state. In other words,

\section{CONCLUSION}

A method of inferring human emotions by stimulating the brain using nature sounds as excitation signals and analyzing and interpreting the variations in the brain waves was presented. In the proposed method, classifying the emotional features of brain waves was replaced with the classifying table instead of the classifiers such as KNN and SVM. This could save time and expenditure because the big data analysis to utilize so-called Artificial Intelligence, Deep Learning, and etc. is not required to perform. Nevertheless, it seems that the performance can be improved by using the classifying table method proposed in this paper and algorithms using artificial intelligence. In order to prove the method proposed in this paper, only one natural sound - the Niagara Falls sound - was selected to examine the possibility, but in the future, it is necessary to investigate many EEG signals by performing stimulation on many different excitation signals.

Nature sounds with the spectrum of pink noise as shown in Fig. 1 show that even though there are relative differences, they generally have a good effect on humans, and generate gamma waves that recognize positive emotions and even happiness. This result shows that the method presented in this paper is useful. In addition, it can be seen that the features of the brain waves used in this paper were also meaningful data.

\section{REFERENCE}

[1] https://thoracickey.com/fundamentals-of-eeg-signalprocessing/ "Fundamentals of EEG Signal Processing, Aug. 2016.

[2] Zhixing Tian, Bong-Young Kim, Myung-Jin Bae. "Study on acoustic analysis of Cleveland Dam waterfall sound". International Journal of Engineering Research and Technology, Volume 13, Issue 6, 2020, Pages 1159-1164.

[3] Ik-Soo Ahn, Bong-young kim, Myung-jin Bae. "A study on the human sensation of the reed wind sound in ASMR". International Journal of Engineering Research and Technology, Volume 12, Issue 9, 2019, Pages 14941499.

[4] Zhixing Tian, Bong-Young Kim, Myung-Jin Bae. "Study on the acoustic characteristics of Sunwapta Falls". International Journal of Engineering Research and Technology, Volume 13, Issue 5, 2020, Pages 962-966.

[5] Kyoung-su Yeo, Myungsook Kim, Myung-Jin Bae. "Acoustic characteristics of the forest sounds inducing sleep. Information (Japan) Volume 18, Issue 10, October 2015, Pages 4407-4412. it can be seen that more $\alpha, \beta$, and $\gamma$-wave are generated in the Near-state and the Far-state than in the Before-state.

The results of this section show that listening to pink noise generally leads to positive emotions, even if there are relative differences. Furthermore, it results in a good emotion to recognize the happiness caused by the $\gamma$-wave occurrence.

[6] Wei-Long Zheng, Jia-Yi Zhu, and Bao-Liang Lu, "Identifying Stable Patterns over Time for Emotion Recognition from EEG", IEEE Transactions on Affective Computing, Vol. 10, No. 3, pp. 417-429, July-Sept. 2019.

[7] Ting-Mei Li, Han-Chieh Chao, and Jianming Zhang, "Emotion classification based on brain wave: a survey", Human-centric Computing and Information Sciences, (https://doi.org/10.1186/s13673-019-0201-x), 2019.

[8] Zeynab Mohammadi, Javad Frounchi, and Mahmood Amiri, "Wavelet-based emotion recognition system using EEG signal", The Neural Computing Applications forum (2017) 28: 1985-1990, (doi 10.1007/s00521-015-2149-8).

[9] Ik-Soo Ahn, Bong-Young Kim, Kwang-Bock You, Myung-Jin Bae "A Study on the Characteristics of an EEG Based on a Singing Bowl's Sound Frequency", Studies in Computational Intelligence Volume 789, 2019, Pages 233-243.

[10] Hyun-ju Lee, Dong-il Shin, and Dong-kyoo Shin, "The Classification Algorithm of Users' Emotion Using BrainWave", J-KICS, Vol. 39C, No. 02, pp. 122-129, Feb. 2014.

[11] Bong-Young Kim, Zhixing Tian, Myung-Jin Bae. "A study on the causes of revenge psychology of Klaxon sound". International Journal of Engineering Research and Technology Volume 12, Issue 12, 2019, Pages 2947 2952.a

[12] Hafeez U. Amin, Aamir S. Malik, Rana F. Ahmad, N. Badruddin, N. Kamel, M. Hussain, and Weng-Tink Chooi, "Feature extraction and classification for EEG signals using wavelet transform and machine learning techniques", Australasian College of Physical Scientists and Engineers in Medicine (2015) 38, pp. 139-149, Feb. 2015.

[13] Wan Amirah, W. Azlan, and Yin Fen Low, "Feature Extraction of EEG Signal - A Review”, IEEE Conference on Biomedical Engineering and Sciences, Dec. 2014.

[14] Semion Kizhner, Thomas P. Flatley, Norden E. Huanh, Karin Blank, and Evette Conwell, "On the Hilbert-Huang Transform Data Processing Systems Development”, 2014 IEEE Aerospace Conference Proceedings, pp. 1961-1979, IEEE, 2014.

[15] Rami J. Oweis and Enas W. Abdulhay, "Seizure classification in EEG signals utilizing Hilbert-Huang transform", http://www.biomedical-engineeringonline.com/content/10/1/38, 2011.

[16] Shin-Ae So, Kang-Hee Lee, Kwang-Bock You, HaYoung Lim, and Ji Su Park, "A Study of Peak Finding Algorithms for the Autocorrelation Function of Speech Signal", Journal of The Korea Society of Computer and Information, Vol. 21, No. 12, PP. 131-137, Dec. 2016 
International Journal of Engineering Research and Technology. ISSN 0974-3154, Volume 13, Number 8 (2020), pp. 1927-1933

(C) International Research Publication House. https://dx.doi.org/10.37624/IJERT/13.8.2020.1927-1933

[17] Kwang-Bock You, Shinae So, and Kang-Hee Lee, "A Comparative Study of the Speech Signal Parameters for the Consonants of Pyongyang and Seoul Dialects Focused on the affricates “/ 不/ ᄎ", Journal of Knowledge Information Technology and Systems, Vol. 14, No. 4, pp. 411-423, Aug. 2019.

[18] Zhixing Tian, Bong-Young Kim, Myung-Jin Bae. "A study on the effect of wearing masks on stress response". International Journal of Engineering Research and Technology, Volume 13, Issue 4, 2020, Pages 807-813.

[19] Marco Weiergraber, Anna Papazoglou, Karl Broich, and Ralf Muller, "Sampling rate, signal bandwidth and related pitfalls in EEG analysis", Journal of Neuroscience Methods 268 (2016), pp. 53-56, May 2016. 